\title{
Wireless Sensor Network Simulation Environment
}

\author{
Taha M. Alfaqih \\ Information System Department \\ King Saud University \\ Riyadh, Saudi Arabia
}

\author{
Abdullah M. Al-Dhelaan \\ Computer Science Deptartment \\ King Saud University \\ Riyadh, Saudi Arabia
}

\author{
Mohammad Mehedi Hassan \\ Information System Department \\ King Saud University \\ Riyadh, Saudi Arabia
}

\begin{abstract}
Abstract-Wireless Sensor networks are infrastructure-less and fully distributed systems of self-configurable and selforganized nodes that are wishing to share data over the air. Nodes could be PCs, mobile phones, printers, etc. The main challenge of sensor networks is its characteristics (dynamic topology, variable capacity, infrastructure less, bandwidth, computing power, battery lifetime, etc.). Another challenge is the deployment operation. In this paper we proposed a method of random deployment (algorithm) and simulation tool to simulate how these sensors work and find the solutions to increase their lifetime, and find the suitable method for deploying sensors in the targeted area (such as isolated area, military area, and in areas infested with terrorist) by using some algorithms that reduce the sensor energy..
\end{abstract}

\section{Keyword}

Wireless Sensor Network, Simulator, Connectivity Hole Problem, Coverage Hole Problem, Uplink Zone.

\section{INTRODUCTION}

Wireless Sensor networks are infrastructure-less and fully distributed systems of self-configurable and self-organized nodes are wishing to share data over the air. Nodes could be PCs, mobile phones, printers, etc. WSN composed of distributive heterogeneous / homogenous sensors which distributed in specific environment to monitor specific phenomena, such as pollution, temperature, pressure, motion, sound or pressure and send their information by the network to a sink, for analysis and take the appropriate decision [1]. The main challenging of sensor networks is its characteristics (dynamic topology, variable capacity, infrastructure less, bandwidth, computing power, battery lifetime, etc.). Another challenge is the deployment operation. WSN deploy in places which are difficult to reach and away from the power source, such as battlefields and volcanic zones [2].

Wireless sensor networks technology become more attracted due the enormous evolution in the micro electro mechanical system (MEMS) [3]. Nowadays, sensors become more low energy consumption and low cost; and the sensor node consist of sensing unit, data processing unit and communicating components. A huge size of sensors node create the networks of sensor. Sensor has an ability to correspond and to communicate with each other [4].

The nature area cause difficulty not only in getting the sensors to replace and recharge the batteries, and the deployment strategy will be achieved by reducing the total number of sensors required. Of this challenge shows two problems: Coverage hole: some areas will be out of overage of the sensor range, and Connectivity hole: some of nodes are disconnected to each other, and cannot reach the sink [5].
The goal of this work is to find the suitable method for deploying sensors in the targeted area (such as isolated area, military area, and in areas infested with terrorist) by using an efficient algorithm that reduces the sensor energy. Finding the suitable method for routing by choosing the suitable routing protocol, and reduce the consumption of sensor's energy to increase the life of wireless sensor network, and to create a simulation for WSN, that will be used as a tool to assist in teaching and achieving an excellent understanding WSN operation and behavior as well as identifying the WSN's capabilities. This work use also to enhance the educational process rather than evaluating the algorithms used in the operation of WSN. It also supports the understanding of computer science students to this important topic.

The rest of the paper is organized as follows. Section2, review the related work. Section 3 gives a simulation method that contain of deployment method, routing constraints, power consumption, and analysis results. Section 4 concludes.

\section{RELATED WORK}

The Wireless Sensor Network simulator is designed by David J. Stein and Esq [6]. The simulation consists of two stages: deploying the network and running simulations. Before deploying the network, the properties of the network such as number of nodes, sensor radius, sensor period, sensor cost, transmitter period, transmit cost, receive cost, directed or random routing should be set using the configuration sliders in the GUI [7].

The deployment method in the simulator is designed by David J. Stein and Esq [6], is dependent on the density of sensors which limited to 400 sensors. This area is fixed in other word it's unchangeable. Whenever the number of sensors is decreased, they will concentrate on specific region within the whole area. Whenever the radius of the sensor is increase or decrease there is no effect on the number of sensors, which cause data redundancy, and loss for the energy of the sensors.

This simulator [6], was written in C\# using Microsoft Visual Studio.NET 2003.

We took some ideas from this simulator, and the method of operation is similar to it, but we added identification number for each sensor to easily control, and identify the position of each sensor and its status (Active or Death). For the method of deployment, there is a developed deployment algorithm, and try to find the suitable number of sensors that will be deployed depending on the interested area and the radius of available sensors, and we added the Gossiping and Flooding for the routing technique. 


\section{SIMULATION METHOD}

The model of WSN proposed simulator Figure [1] which uses different metrics restrictions, such as communication range, power consumption, sensor radius, routing techniques and the interested area that to be deployment of sensors.

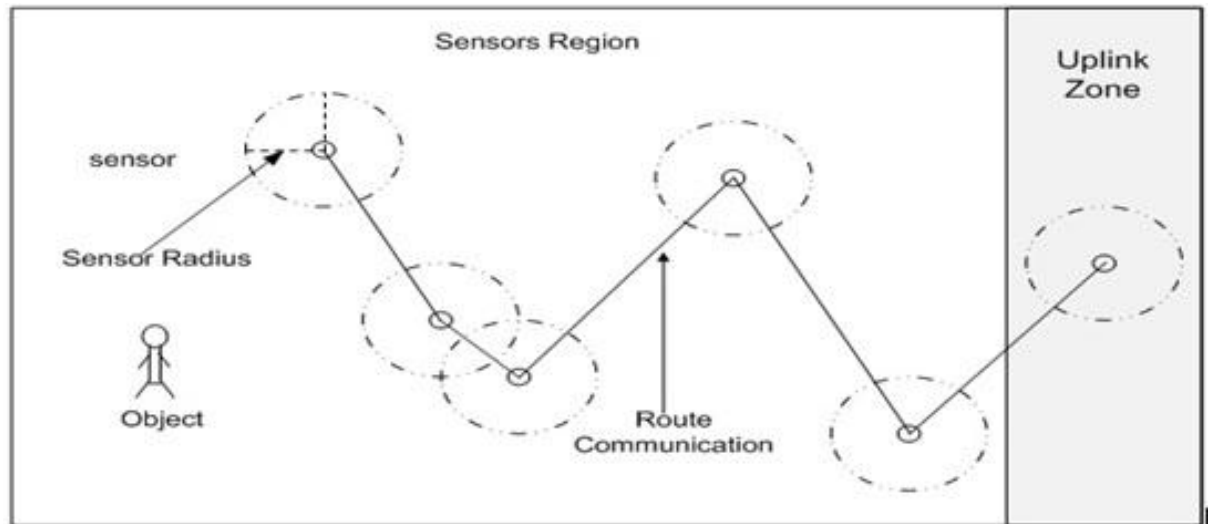

Fig 1: WSN Simulator Model

In this model we assume that: each sensor has unique id, radius for sensing, communication radius, and initial power, region has a coordinate, and also each sensor belong to one region.

\subsection{Simulator Design}

In the proposed simulator, we start the deployment of sensors process, we first must set the parameters for sensors to be deployed, including the distance that sensors will be deployed in. The number of sensors that will be deployed in this area will be calculated by using specific equation, so the number of nodes must be suitable and covered this entire region. The parameters that will be used in the proposed simulator are as follows.

Size of network, Sensor range, Detection delay, Detection energy, Detection energy, Transmission time, Transmission energy, receiving energy.

The parameter must be consider when choosing the sensors, in addition there are many attributes for sensor, and these are: sensor Id, Position, Power Level, and Status.

Sensors are deployed according to the area to be monitored. The number of sensors is calculated of the network size based on this area, taking into account the radius of each sensor (We proposed the deployment algorithm) in the section 3.2.

The sensor Id is represented of identifier to distinguish a node, position is X-Y Coordinate system of nodes position in the area of deployment, and power level indicates the available power for the current node, and the current state of the individual node, is active or death (become death when the power is not enough to do any task for the sensor, sensing, computation and communication).

This simulator designed for a flexible used, enables the user to enter the parameters for the sensors, such as: Communication Range, Sensor Radius, Sensor Period and other parameters.

\subsection{Deployment Method}

To obtain the suitable number of sensors to covered interested area, suppose we want to monitor area (Width * Length), we calculate the number of sensor as following:
Where $\mathrm{A}=$ Width*Length, and $\mathrm{R}$ is a radius of the sensor.

The deployment strategy is randomly based on Random Algorithm:

Random_deployment ( ) \{

List_Sensor[i];

While (Count (List_Sensor[i] < NetworkSize) \{

While (Count (List_Sensor[i] < NetworkSize)

$\{\operatorname{Node}(\mathrm{X}, \mathrm{Y})$

While (i< Count (List_Sensor[i])

If (Sensor(X, i) $>$ New_Sensor(X, i))

Insert (New_Sensor(X, i), List_Sensor[i]);

$$
\mathrm{I}+1 ;\}
$$

If ( $\mathrm{i}=$ List_Sensor[i])

Insert (New_Sensor(X, i), List_Sensor[i]) ;\}\}

There are two major problems that we encountered during the random deployment of the sensors: Coverage hole problem and Connectivity hole problem as shown in Figures [2] and [3]. 
The Coverage hole problem is exists, but a few percentage. The second problem which is Connectivity hole problem is almost non-existent and very smaller than the previous one.

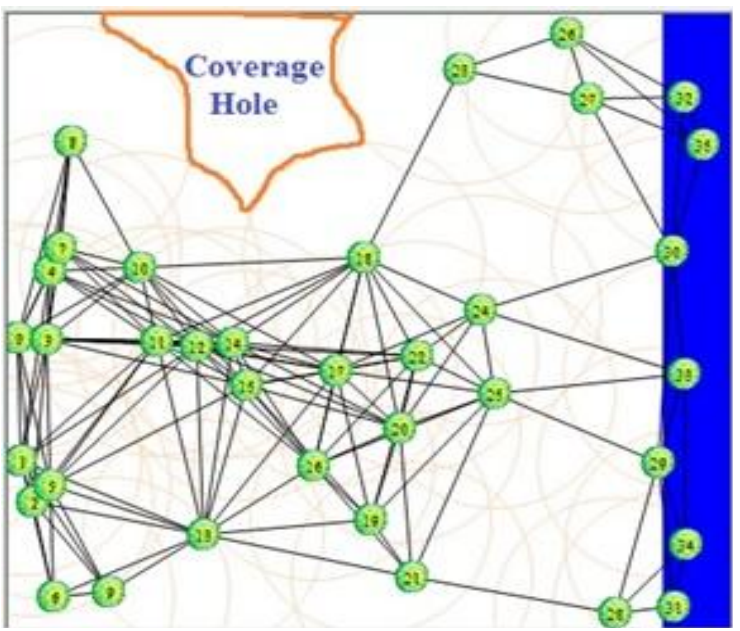

Fig2 : Coverage Hole Problem

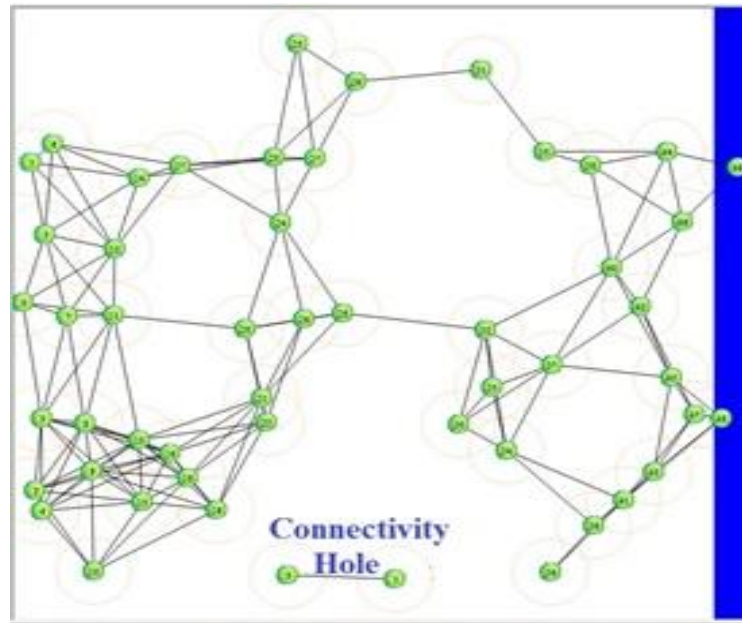

Fig3: Connectivity Hole Problem

We reduce these problems, by computing the suitable numbers of sensors depend on required area to be monitor, and sensor's radius. Taking into account that the sensor's radius will reduce the coverage hole problem. Addition the transmission radius to reduce the connectivity hole problem.

We designed this simulator to allow multiple methods of setting the number of sensors, automatically and manually. In the automatic method the simulator will compute the number of sensors depending on the entered area and sensor's radius.

In the Other method the user can set any number of sensors manually to be deployment.

Figures [4] and [5] illustrate the deployment of different sensors numbers depending on the required area and the sensor's radius. Whenever the sensor's radius was large, the required number of sensors will be few, and the coverage for the area is better. For example, in Figure [4] the area to be monitored about $2 \mathrm{~km}$, and a sensor's radius is about $10 \mathrm{~m}$, the suitable number of sensors required to cover this area is 20 sensors. While in the Figure [5], we notice the same area, but the sensors radius is $5 \mathrm{~m}$, so it requires double number of sensors which equals to 80 sensors, and so on for the others of Figures [6] [7].

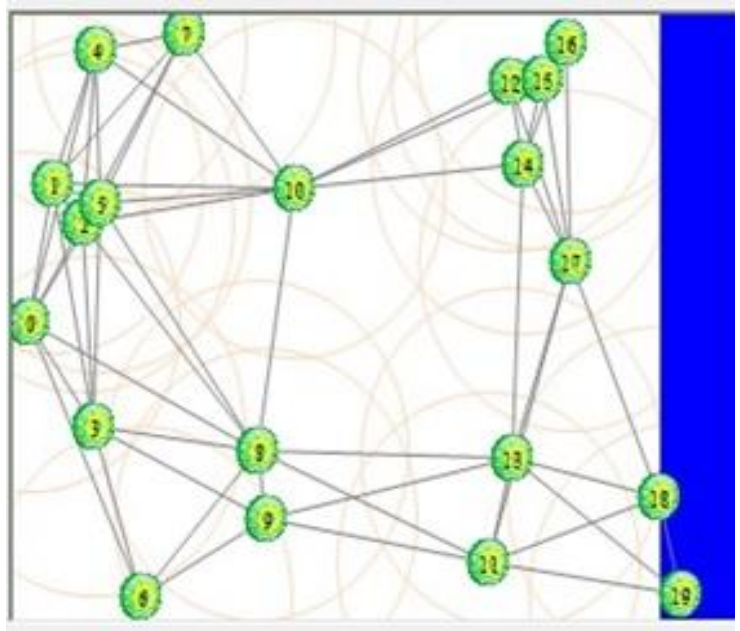

Fig4: Area $=2 K$, Radius $=10$, NetSize $=20$

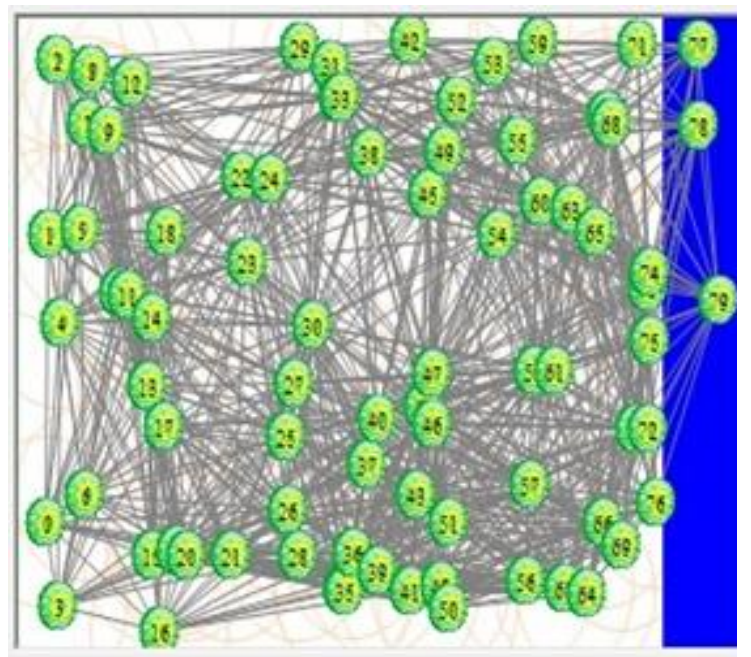

Fig5: Area=2K, Radius=5, NetSize $=80$

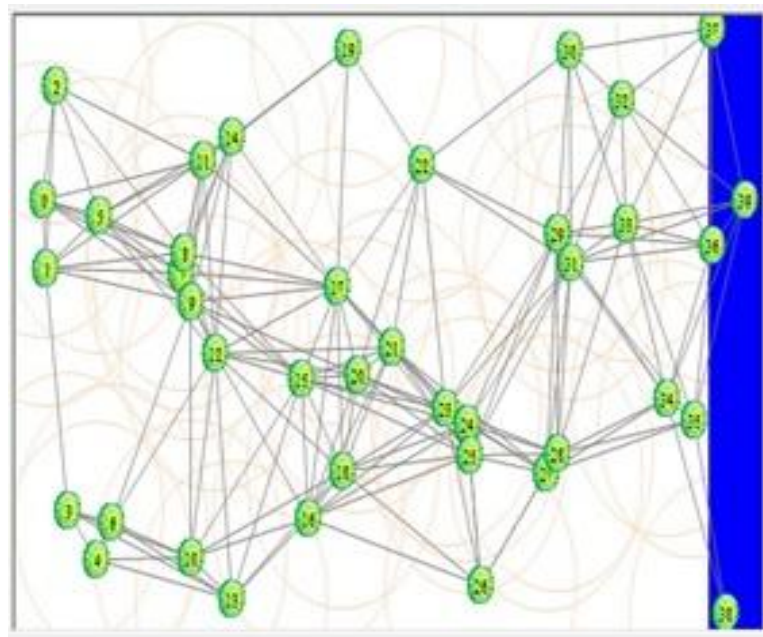

Fig6: Area $=4 \mathrm{~K}$, Radius $=10$, NetSize $=40$ 


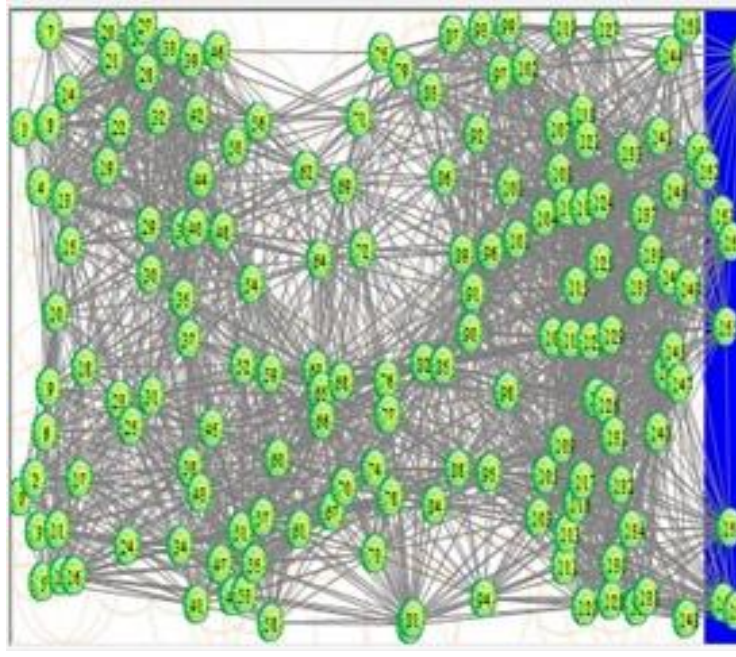

Fig7: Area=4K, Radius=5, NetSize $=160$

\subsection{Routing Constraints}

Wireless Sensor Network routing is varying from routing in other tradition network such as computer network. And the WSN topologies are dynamic, and it can exist in different stages. In this simulation we assume that the nodes are fixed in their locations, and the deployment was a random method. We use the some methods to route data packets from the sensors field to the uplink zone (Base Station).These methods are: Gossiping technique, flooding technique and also we used the random technique, which is used in [5].

\subsubsection{Gossiping Technique}

Gossiping technique [4], uses directed algorithm, schemes which distribute the computational burden and in which a node communicates with a randomly chosen neighbor [8]. Message is deployed from sink or a node, and select a nearest neighbor node to be the beginning of the route of the message, when this node is receiving the message, the node is verifies the intended receiver, if it is intended to receive this message, the routing will stop there, and if they are not the intended receiver, then it chooses a neighbor node and send the message to this neighbor, Thus the process is repeated in recursive structure [9].

This process, maintain the list of all nodes that have been visited and this ensures that the route of the message will not remain in an infinite loop.

The gossiping technique depends on some routing parameters which such as Residual Energy: The emphasis placed on the residual energy of the nodes in making routing decisions. Initial Energy: The emphasis placed on the initial energy of the nodes in making routing decisions and Routing Delay: The amount of time between packet-routing decision updates, and Communication Cost [4] [10].

\subsubsection{Flooding Technique}

The flooding protocol [4] uses a traditional method to deploy the data packets. In the flooding model, the packet control is sent from the sink or a node to each node that is neighbor of this node. Any node receives this packet send a message to all its neighboring nodes including this node. This process is repeated in all nodes that receive the message, causing repeated data in the nodes and increases packet collision packets problem [8].

Therefore only one knows all information sent message is the intended receiver. This information contains delivery status "successful". The other nodes continue sending the same message to other nodes, and this process is repeated an infinite loop [3] [11].

In this process, maintain the list of all visited nodes that received the packets, and this ensures that the route of the message will not remain in an infinite loop

\subsection{Power Consumption}

The main challenge in WSN is the power consumption, and it considered one of the main aims for the researchers. They made a lot of efforts to increase the life time of sensors. As we know the energy management in a WSN has essential effect on the network life time [10].

As matter of fact the depletion of the sensors power occur during the main sensors activities such as (sensing, computation and communication process).

The main consumption for the energy is the communication process between sensors and the sensing process too.

The following equations show how to calculate the power consumption in sensors, which have been used in this simulator:

Power $=($ Power $)-($ Detection_Energy $) . \quad$ Eq.2

Power $=($ Power $)-($ Transmission_Energy_Per_Msg. $) \quad$ Eq.3

Power $=($ Power $)-($ Receiving_Energy_Per_Msg. $) \quad$ Eq.4

Where Eq.2, demonstrates how to calculate the energy consumption per node during the sensing process events at the time of the node activation. Eq.3 and Eq.4 illustrate how the energy consumption, is calculated at each node per message sent or received.

The sensors power consumption is different from one type to another. In our simulator, the user is enabled to enter values of energy consumption based on the specifications of the sensor available to him. These values are set in these parameters (detection cost, transmission cost, receiving cost).

Another process of the power consumption is the computation process (processing). The computation process is an important process, but the energy consumed in this process is a little. So we merge with the sensing process parameter with the computation process parameter in our simulator. Due the user can enter the value of this process with the value of sensing parameter.

Figures [8] [9], are examples to illustrate the process of power consumption in the simulator. In Figure [8], there is an illustration for the beginning of monitoring process. Field power level shows how much energy each sensor consumed, and the status of this sensor is active as shown in the field of status. The value is sequentially until become zero. When the value is equal to zero, it indicates that the sensor cannot operate any more (Death), as shown in Figure [9] become death when the power is not enough to do any task for the sensor, sensing, computation and communication.

\begin{tabular}{|c|c|c|c|}
\hline NodeID & Position & PowerLevel & Status \\
\hline 1 & $(18,275)$ & 974 & Active \\
\hline 2 & $(24,431)$ & 982 & Active \\
\hline 3 & $(27,301)$ & 966 & Active \\
\hline
\end{tabular}




\begin{tabular}{|l|c|l|l|}
4 & $(30,169)$ & 976 & Active \\
\hline 5 & $(41,534)$ & 966 & Active \\
\hline 6 & $(45,44)$ & 976 & Active \\
\hline 7 & $(49,136)$ & 978 & Active \\
\hline 8 & $(52,277)$ & 958 & Active \\
\hline 9 & $(54,118)$ & 970 & Active \\
\hline 10 & $(60,557)$ & 956 & Active \\
\hline 11 & $(73,38)$ & 972 & Active \\
\hline 12 & $(74,410)$ & 966 & Active \\
\hline 13 & $(89,172)$ & 948 & Active \\
\hline 14 & $(90,531)$ & 942 & Active \\
\hline 15 & $(92,30)$ & 940 & Active \\
\hline
\end{tabular}

Tabel.1: Example of power Consumption

\begin{tabular}{|c|c|c|c|}
\hline NodeID & Position & PowerLevel & Status \\
\hline 80 & $(18,275)$ & 974 & Active \\
\hline 79 & $(24,431)$ & 982 & Active \\
\hline 78 & $(27,301)$ & 966 & Death \\
\hline 77 & $(30,169)$ & 976 & Active \\
\hline 76 & $(41,534)$ & 966 & Death \\
\hline 75 & $(45,44)$ & 976 & Death \\
\hline 74 & $(49,136)$ & 978 & Death \\
\hline 73 & $(52,277)$ & 958 & Death \\
\hline 72 & $(54,118)$ & 970 & Death \\
\hline 71 & $(60,557)$ & 956 & Death \\
\hline 70 & $(73,38)$ & 972 & Active \\
\hline 69 & $(74,410)$ & 966 & Death \\
\hline 68 & $(89,172)$ & 948 & Active \\
\hline 67 & $(90,531)$ & 942 & Death \\
\hline
\end{tabular}

Tabel.2: Example of power Consumption

\subsubsection{Analysis and Results}

In this section, we view some of the results that was appeared during the simulation process with our different assumption and setting for the sensor parameters. These setting and assumption were divided into four stages. In each stage, the values of parameter changed depending on their effect on the sensors operation and power consumption, such as Sensors radius and Communication radius. Also other an assumptions that are mentioned in each stages.

Stage1: When we assume: Sensor's Radius $=10$, Communication Radius $=10$, Cost of Detection $=2 \mathrm{~dB}$, Cost of Sending $=5 \mathrm{~dB}$

Cost of receiving $=2 \mathrm{~dB}$, Initial Power $=1000 \mathrm{~dB}$. Where Connectivity Problem (Conn.Pro), Coverage Problem (Cav.Pro).See Table 3, Power Consumption Average (Avg.PC). Average time for the simulation $=2$ minutes.

\begin{tabular}{|c|c|c|c|c|}
\hline Area & Net.Size & Conn.Pro & Cav.Pro & $\begin{array}{c}\text { Avg.PC } \\
\%\end{array}$ \\
\hline $2 \mathrm{~K}$ & 20 & 0 & $(1-5 \%)$ & $53 \%$ \\
\hline $4 \mathrm{~K}$ & 40 & 0 & $(1-5 \%)$ & $34 \%$ \\
\hline $6 \mathrm{~K}$ & 60 & 0 & $(6-10 \%)$ & $35 \%$ \\
\hline $8 \mathrm{~K}$ & 80 & 0 & $(1-5 \%)$ & $35 \%$ \\
\hline $10 \mathrm{~K}$ & 100 & 0 & $(1-5 \%)$ & $34 \%$ \\
\hline
\end{tabular}

Table 3: Illustrates percentage of coverage problem, connectivity problem and power consumption in different interest areas at a specific time.

We proposed classification of coverage problem to percentages. Where we consider the percentage that between 1 and 5 is the problem in safe mode, and the percentage between 6 and 10 is small problem can be overlooked, where the percentage rate between 11 and 15 is considered medium problem.

We have sought to minimize them, we will observe in the cases studied, and the percentage of coverage between 16 and 20 is considered a big problem, and we sought to minimize of them as we will see in the cases that have been studied also. We conclude our idea is success compared to what has been studied in [6].

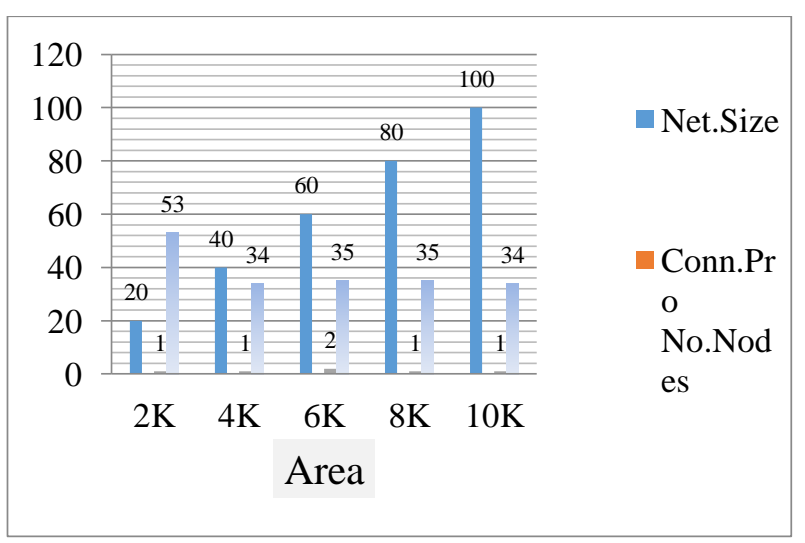

Fig. 8: Representation of the data mentioned in Table 3.

The coverage problem which shown in the Table 4, appeared when the area is $6 \mathrm{~km}$. On the other hand, the energy consumed relatively little except when the intended area is 2 $\mathrm{km}$. In this case energy consumption reach to $53 \%$, and the connectivity Problem isn't appear.

Note: If the percentage of the coverage problem is between 1 and $5 \%$ considered to be in safe mode.

Stage 2: When we assume: Sensor's Radius $=20 \mathrm{~m}$, Communication Radius $=10 \mathrm{~m}$, Cost of Detection $=2 \mathrm{~dB}$, Cost of Sending $=5 \mathrm{~dB}$, Cost of receiving $=2 \mathrm{~dB}$, Initial Power $=1000 \mathrm{~dB}$

In the Table.5, we notice that when we increased the sensors radius the power consumption is increased, also the coverage problem is increased, which a reached to $(11-15 \%)$, due to the number of sensors is decreased to $25 \%$ of what it was in the first stage, when the sensors radius $=20$. On the other hand, the energy consumption increased significantly, according to the connectivity problem, we noticed that there is only one state that appeared in area $=10 \mathrm{Km}$. 


\begin{tabular}{|c|c|c|c|c|}
\hline Area & Net.Size & Conn.Pro & Cav.Pro & $\begin{array}{c}\text { Avg.PC } \\
\%\end{array}$ \\
\hline $2 \mathrm{~K}$ & 5 & 0 & $(1-5 \%)$ & $88 \%$ \\
\hline $4 \mathrm{~K}$ & 10 & 0 & $(6-10 \%)$ & $65 \%$ \\
\hline $6 \mathrm{~K}$ & 15 & 0 & $(6-10 \%)$ & $55 \%$ \\
\hline $8 \mathrm{~K}$ & 20 & 0 & $(11-15 \%)$ & $55 \%$ \\
\hline $10 \mathrm{~K}$ & 25 & 1 & $(11-15 \%)$ & $41 \%$ \\
\hline
\end{tabular}

Table 4. Illustrates percentage of coverage problem, connectivity problem and power consumption in different interest areas at a specific time, according to the assumptions that are mentioned above.

The figure [9], represented the data mentioned in Table [4].

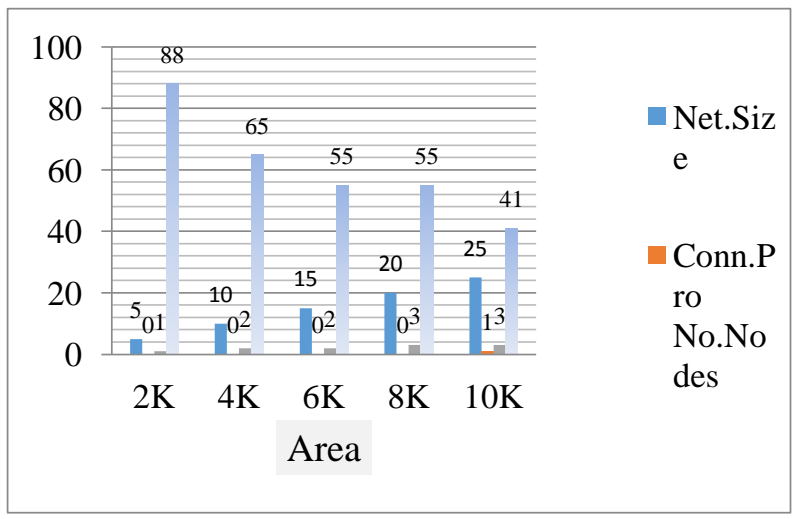

Fig.9: Representation of the data mentioned in Table 4.

Stage 3: When we assume: Sensor's Radius $=10$, Communication Radius $=20$, Cost of Detection $=2 \mathrm{~dB}$, Cost of Sending $=5 \mathrm{~dB}$, Cost of receiving $=2 \mathrm{~dB}$, Initial Power $=1000 \mathrm{~dB}$.

In the Table 5, we notice that the coverage problem doesn't exist, due the communication radius of sensors is increased, when the communication radius $=20$.Also the consumed energy is less than what it was in the table 5(Stage 2). Because of the reduction in the radius of sensors .The problem of connectivity became entirely absent, due to increasing of communication radius of sensors.

\begin{tabular}{|c|c|c|c|c|}
\hline Area & Net.Size & Conn.Pro & Cav.Pro & $\begin{array}{c}\text { Avg.PC } \\
\%\end{array}$ \\
\hline $2 \mathrm{~K}$ & 5 & 0 & $(1-5 \%)$ & $91 \%$ \\
\hline $4 \mathrm{~K}$ & 10 & 0 & $(1-5 \%)$ & $72 \%$ \\
\hline $6 \mathrm{~K}$ & 15 & 0 & $(1-5 \%)$ & $58 \%$ \\
\hline $8 \mathrm{~K}$ & 20 & 0 & $(1-5 \%)$ & $47 \%$ \\
\hline $10 \mathrm{~K}$ & 25 & 0 & $(1-5 \%)$ & $37 \%$ \\
\hline
\end{tabular}

Table 5. Illustrates percentage of coverage problem, connectivity problem and power consumption in different interest areas at a specific time.

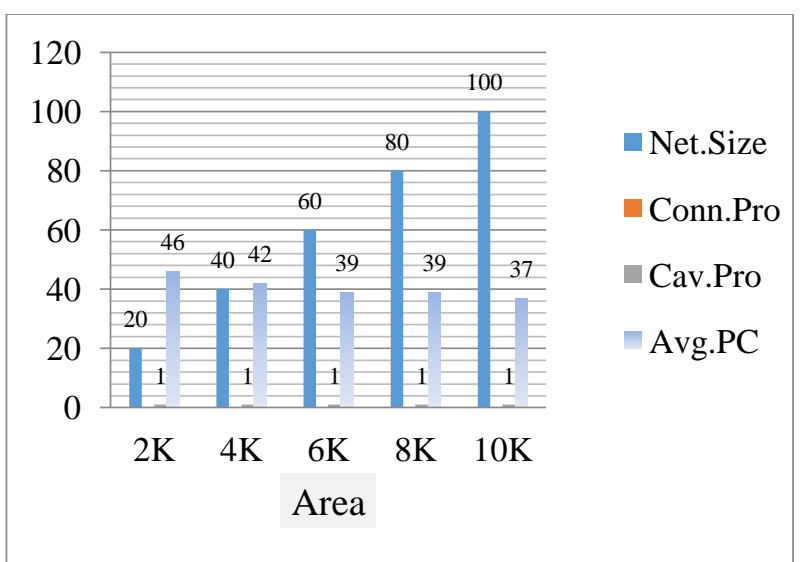

Fig.10 Representation of the data mentioned in Table 5.

Stage 4: When we assume: Sensor's Radius $=20$, Communication Radius $=20$, Cost of Detection $=2 \mathrm{~dB}$, Cost of Sending $=5 \mathrm{~dB}$, Cost of receiving $=2 \mathrm{~dB}$, Initial Power $=1000 \mathrm{~dB}$.

In the Table 6, we notice that when we increased sensors radiuses and communication radius of sensors, the power consumption is significantly increased. On the other hand we notice that the coverage problem and connectivity problem became in entirely absent.

\begin{tabular}{|c|c|c|c|c|}
\hline Area & Net.Size & Conn.Pro & Cav.Pro & $\begin{array}{c}\text { Avg.PC } \\
\%\end{array}$ \\
\hline $2 \mathrm{~K}$ & 20 & 0 & $(1-5 \%)$ & $53 \%$ \\
\hline $4 \mathrm{~K}$ & 40 & 0 & $(1-5 \%)$ & $34 \%$ \\
\hline $6 \mathrm{~K}$ & 60 & 0 & $(6-10 \%)$ & $35 \%$ \\
\hline $8 \mathrm{~K}$ & 80 & 0 & $(1-5 \%)$ & $35 \%$ \\
\hline $10 \mathrm{~K}$ & 100 & 0 & $(1-5 \%)$ & $34 \%$ \\
\hline
\end{tabular}

Table 6. Illustrates percentage of coverage problem, connectivity problem and power consumption in different interest areas at a specific time.

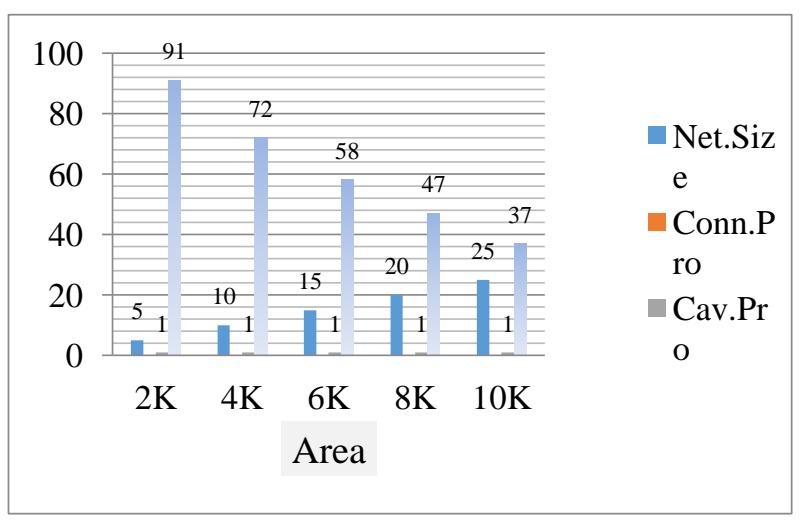

Fig.11: Representation of the data mentioned in Table 6

From the study of the previous stages, we found that the best settings for the sensor networks is the stage 3, by set the radius of sensors $=10$, and communication radius $=20$, in which is reduces the connectivity problems, coverage hole problem and energy consumption are reduced. 
Through our work in the simulator, we found that the sensor that nearer to the uplink zone consume large amount of energy compared with the other sensors, so these sensors die before other sensors in the interested area, due to these sensors almost all the time in activity .

\section{CONCULASION}

The wireless sensor networks are set of sensors that work together to form network. The main challenging of sensor networks is its characteristics (dynamic topology, variable capacity, infrastructure less, bandwidth, computing power, battery lifetime, etc.). Another challenge is the deployment operation.

In this paper we are designed and implemented a simulation tool to simulate how these sensors work and to find the suitable solutions to increase the coverage and the sensors lifetime.

We have created a virtual space in which the sensors are randomly deployed. This area will be considered as a battlefield that needs suitable number of sensors. The number of sensors that is needed for this area will be calculated using specific formula.

The main objective of this project is to enhance educational process, but not to evaluate the algorithms used in the operation of WSN. It also increase concept of computer science for students in this important topic.

In this simulator, the number of sensors that the users or students can be deployed, is not exceeding 500 sensors. The needed number of sensors that which be deployed can be sent using automatically or manually .In the automatically way ,the user selecting the area to be monitored, in this case the simulator automatically determines the number of sensors depending on the area that entered and the sensors radius. In the second way, the user will enter the number of sensors to be scattered manually.

After that, the user selects network features a (Communication radius, Sensor radius, Detection data), as well as the user can choose from one species Routing Technique (Random, Gossiping, Flooding). In the Random routing technique, choosing randomly from sensing field to the uplink zone (base station), each node select nearest neighbor to deliver data packet until reach to the target. The Gossiping technique is "schemes which distribute the computational burden and in which a node communicates with a randomly chosen neighbor" [8].

Flooding technique, the data will be broadcasted from the source node to all its neighbor nodes and the neighbor will repeat the same action. Which what we call bottleneck in the network. Due to the huge amount of redundant data [4].

In this simulator, we notice that the number of sensors depend on the area and the radius of sensors and the Communication Range. For example, whenever the sensors radius is increased the number of deployed sensors is decreased; in this case the power consumption is increased. Using this scenario leads to reduce the problem of Connection hole and Connectivity Hole.

The energy will be consumed during the sensors operation such as sending, sensing and receiving. It will also depend on the protocol used for data transfer [10]

\section{REFERENCES}

[1] J. Zheng and A. Jamalipour, Wireless sensor networks: a networking perspective: John Wiley \& Sons, 2009.

[2] M. Younis, M. Youssef, and K. Arisha, "Energy-aware routing in cluster-based sensor networks," in Modeling, Analysis and Simulation of Computer and Telecommunications Systems, 2002. MASCOTS 2002. Proceedings. 10th IEEE International Symposium on, 2002, pp. 129-136.

[3] M. M. Hassan, B. Song, and E.-N. Huh, "A framework of sensor-cloud integration opportunities and challenges," in Proceedings of the $3 \mathrm{rd}$ international conference on Ubiquitous information management and communication, 2009, pp. 618-626.

[4] K. Akkaya and M. Younis, "A survey on routing protocols for wireless sensor networks," Ad hoc networks, vol. 3, pp. 325-349, 2005.

[5] A. A. Abbasi and M. Younis, "A survey on clustering algorithms for wireless sensor networks," Computer communications, vol. 30, pp. 2826-2841, 2007.

[6] E. David J. Stein, "Wireless Sensor Network Simulator v1.1," Application, 2005.

[7] H. K. Kalita, "A new algorithm for end to end security of data in a secure self organized wireless sensor network," Journal of Global Research in Computer Science, vol. 1, 2010.

[8] S. Boyd, A. Ghosh, B. Prabhakar, and D. Shah, "Randomized gossip algorithms," Information Theory, IEEE Transactions on, vol. 52, pp. 2508-2530, 2006.

[9] H. K. Kalita and A. Kar, "Simulator based performance analysis of Wireless Sensor Network-A new approach," in Wireless and Mobile Computing, Networking and Communications (WiMob), 2011 IEEE 7th International Conference on, 2011, pp. 461-465.

[10] Y. Xu, J. Heidemann, and D. Estrin, "Geographyinformed energy conservation for ad hoc routing," in Proceedings of the 7th annual international conference on Mobile computing and networking, 2001, pp. 70-84.

[11] B. An and S. Papavassiliou, "A mobility-based clustering approach to support mobility management and multicast routing in mobile ad-hoc wireless networks," International Journal of Network Management, vol. 11, pp. 387-395, 2001. 